\title{
S100A8 Enhances IL-1 $\beta$ Production from Nasal Epithelial Cells in Eosinophilic Chronic Rhinosinusitis
}

\section{Ayaka Nakatani}

Department of Otorhinolaryngology-Head and Neck Surgery, Osaka University Graduate School of Medicine

\section{Takeshi Tsuda}

Department of Otorhinolaryngology-Head and Neck Surgery, Osaka University Graduate School of Medicine

\section{Yohei Maeda ( $\nabla$ ymaeda@ent.med.osaka-u.ac.jp )}

Department of Otorhinolaryngology-Head and Neck Surgery, Osaka University Graduate School of Medicine

\section{Masaki Hayama}

Department of Otorhinolaryngology-Head and Neck Surgery, Osaka University Graduate School of Medicine

\section{Daisuke Okuzaki}

Genome information research center, Research institute for microbial diseases, Osaka university

\section{Sho Obata}

Department of Otorhinolaryngology-Head and Neck Surgery, Osaka University Graduate School of Medicine

\section{Toshihiro Kishikawa}

Department of Otorhinolaryngology-Head and Neck Surgery, Osaka University Graduate School of Medicine

\section{Kazuya Takeda}

Department of Otorhinolaryngology-Head and Neck Surgery, Osaka University Graduate School of Medicine

\section{Hidenori Inohara}

Department of Otorhinolaryngology-Head and Neck Surgery, Osaka University Graduate School of Medicine

\section{Research Article}

Keywords: ECRS, nasal polyps, S100, S100A8, IL-1 $\beta$ 
Posted Date: October 1st, 2021

DOI: https://doi.org/10.21203/rs.3.rs-934588/v1

License: (c) (i) This work is licensed under a Creative Commons Attribution 4.0 International License. Read Full License 


\section{S100A8 Enhances IL-1ß Production from Nasal Epithelial Cells in Eosinophilic}

Chronic Rhinosinusitis

\section{Authors:}

Ayaka Nakatani, $\mathrm{MD}^{1 \dagger}$, Takeshi Tsuda MD, $\mathrm{PhD}^{1,2 \dagger}$, Yohei Maeda, $\mathrm{MD}, \mathrm{PhD}^{1^{*} \text {, }}$ Masaki Hayama, MD, PhD ${ }^{1}$, Daisuke Okuzaki, $\mathrm{PhD}^{3}$, Sho Obata, MD ${ }^{1}$, Toshihiro Kishikawa, $\mathrm{MD}, \mathrm{PhD}^{1}$, Kazuya Takeda, $\mathrm{MD}, \mathrm{PhD}^{1,4}$, Hidenori Inohara, $\mathrm{MD}, \mathrm{PhD}^{1}$

${ }^{1}$ Department of Otorhinolaryngology-Head and Neck Surgery, Osaka University Graduate School of Medicine, Suita City, Osaka, Japan

${ }^{2}$ Department of Otorhinolaryngology, Osaka National hospital, Osaka city, Osaka, Japan

${ }^{3}$ Genome information research center, Research institute for microbial diseases, Osaka university, Suita, Osaka, Japan

${ }^{4}$ Department of Otolaryngology, Kindai University, Faculty of medicine, Osaka-Sayama City, Osaka, Japan

$\dagger$ These authors contributed equally to this work

\section{*Corresponding author:}

Yohei Maeda MD, PhD 
Assistant Professor

Department of Otorhinolaryngology-Head and Neck Surgery

Osaka University Graduate School of Medicine

2-2 Yamada-oka, Suita, Osaka 565-0871, Japan

Tel: $+81-6-6879-3833$

Fax: +81-6-6879-3839

E-mail: ymaeda@ent.med.osaka-u.ac.jp

Keywords: ECRS, nasal polyps, S100, S100A8, IL-1 $\beta$

\section{Conflict of Interest}

There are no conflicts of interest to declare.

\section{Authors Contributions}

YM and HI supervised the project. AN and TT analyzed the data and wrote the manuscript. SO, MH, YM, AN, and KT recruited and clinically characterized patients. DO and TK provided advice on project planning and data interpretation. All authors participated in discussion of the results and critically reviewed and approved the final draft.

\section{Funding}

This work was supported by grants from MEXT/JPS KAKENHI grant number T20K182520 (to AN) and T17K113590, T20K097110 (to YM).

\section{Acknowledgments}

We thank Zenis (https://www.zenis.co.jp/editing/index.html) for English language editing. 


\begin{abstract}
Chronic rhinosinusitis is classified into eosinophilic chronic rhinosinusitis (ECRS) and non-eosinophilic chronic rhinosinusitis (NECRS). ECRS is a refractory allergic disease involving a variety of immune and epithelial cells. S100A8 is a damage-associated molecular pattern that is closely related to allergic inflammation. However, the pathological implications of S100A8 in ECRS have not been clarified. We evaluated the role of S100A8 in the pathogenesis of ECRS. Gene expression profiles of nasal polyps obtained from patients with ECRS or NECRS were evaluated using RNA sequencing. S100A8 was identified as a significantly upregulated gene in nasal polyps associated with ECRS. Immunohistochemistry consistently revealed intense S100A8 staining in nasal polyps from patients with ECRS. Human nasal epithelial cells expressed the receptor for advanced glycation end products and Toll-like receptor 4. Recombinant $\mathrm{S} 100 \mathrm{~A} 8$ protein induced interleukin- $1 \beta$ secretion in human nasal epithelial cells. Our data demonstrate that $\mathrm{S} 100 \mathrm{~A} 8$ results in production of interleukin-1 $\beta$ in the nasal epithelium, which may be involved in the pathogenesis of ECRS.
\end{abstract}




\section{INTRODUCTION}

Chronic rhinosinusitis (CRS) is an airway disease with paranasal sinus inflammation. In East Asia, the phenotypes of CRS are heterogeneous, and CRS is subdivided in two groups according to Japanese clinical criteria: eosinophilic chronic rhinosinusitis (ECRS) and non-eosinophilic chronic rhinosinusitis (NECRS) ${ }^{12} 3$. ECRS is an allergic disease that causes olfactory disturbances, nasal obstruction, and nasal discharge. In addition, bronchial asthma is a common complication of ECRS, which is designated as an intractable disease in Japan. ECRS is a type 2 inflammatory disease involving various types of immune cells. Recent studies have shown that the most severe forms of eosinophilic inflammation, including ECRS, are caused by inflammation resulting from a combination of type 2 and type 3 cytokines ${ }^{45}$. The main treatments for ECRS are endoscopic sinus surgery (ESS) and systemic glucocorticoids. However, ESS has a high relapse rate, while long-term systemic glucocorticoid steroid therapy is associated with side effects. Therefore, molecularly targeted therapy based on an understanding of pathogenesis is an attractive alternative for treatment of ECRS.

Nasal epithelial cells are the interface between the host and the environment and act as the first defense mechanism. Nasal epithelial cells produce a variety of cytokines and matrix metalloproteinases that exacerbate allergic inflammation ${ }^{67}$. In particular, when cellular damage occurs, nasal epithelial cells release damage-associated molecular patterns (DAMPs). DAMPs contribute to the activation of various immune cells by type 2 and type 3 cytokines, and previous reports have suggested a relationship between DAMPs and allergic diseases. High mobility group box-1 (HMGB-1), a representative DAMP, enhances interleukin-33 production from nasal epithelial cells ${ }^{8}$. However, the pathological implications of other DAMPs in CRS have not been sufficiently clarified.

$\mathrm{S} 100$ proteins are a group of small $\mathrm{Ca}^{2+}$-binding modulator proteins. Recent work has revealed that $\mathrm{S} 100$ proteins are involved in tumor metastasis and immune responses ${ }^{9}$. In particular, S100A8 protein binds to Toll-like receptor 4 (TLR4) or the receptor for advanced glycation end products (RAGE) and initiates the activation of various immune cells and the production of reactive oxygen species and nitric oxide, contributing to the exacerbation of chronic inflammation 10111213 .

In this study, we attempted to discover the genes involved in the pathogenesis of ECRS via RNA sequencing. We found that $S 100 A 8$ gene expression is upregulated in 
nasal polyps obtained from patients with ECRS. Thus, we sought to clarify the mechanism of ECRS by characterizing the effects of S100A8 on the nasal epithelium.

\section{MATERIALS AND METHODS}

Human subjects

Paranasal sinus tissue samples were collected from seven patients with chronic rhinosinusitis treated between 2014 and 2017 at the Department of Otorhinolaryngology Head and Neck Surgery, Osaka University Hospital. This study was approved by the ethics committee of Osaka University (No. 14463). Written informed consent was obtained from all subjects. CRS was defined according to the guidelines in the European Position Paper on Rhinosinusitis and Nasal Polyps ${ }^{14}$. Patients with ECRS were diagnosed according to criteria established by the Japanese Epidemiological Survey of Refractory Eosinophilic Chronic Rhinosinusitis ${ }^{2}$.

RNA sequencing ( $\mathrm{n}=8$ ECRS, $\mathrm{n}=2$ NECRS), reverse transcription quantitative polymerase chain reaction (RT-qPCR) $(\mathrm{n}=8$ ECRS, $\mathrm{n}=4$ NECRS $)$, and immunohistochemistry (S100A8: $\mathrm{n}=15$ ECRS, $\mathrm{n}=15$ NECRS; RAGE, TLR4, IL1- $\beta$ : $\mathrm{n}$ $=5$ ECRS, $\mathrm{n}=5$ NECRS) were performed.

reverse transcription quantitative polymerase chain reaction (RT-qPCR) and immunohistochemistry experiments were performed three times.

RNA sequencing

Total RNA extracted from nasal polyps from two patients with ECRS and two patients with NECRS were evaluated using RNA sequencing. Library preparation was performed using the SMARTer ${ }^{\circledR}$ Stranded Total RNA Sample Prep Kit-Pico Input Mammalian (TaKaRa, Osaka, Japan). Sequencing was performed on an Illumina HiSeq 2500 platform in 75-base single-end mode. Illumina CASAVA 1.8.2 software was used for base calling. Sequenced reads were mapped to the human reference genome sequence (hg 19) using TopHat version 2.0.13 in combination with Bowtie2 version 2.2.3 and SAMtools version 0.1 .19 . The number of fragments per kilobase of exon per million mapped fragments (FPKMs) was calculated using Cufflinks version 2.2.1 The raw data have been deposited in the NCBI Gene Expression Omnibus database GSE172305). 
RNA extraction and reverse transcription

RNA samples from tissue samples were extracted using TRIzol reagent (Invitrogen, Waltham, MA, USA). Complementary DNA (cDNA) was synthesized using the ReverTra Ace qPCR RT Master Mix (TOYOBO, Osaka, Japan).

RT-qPCR

cDNA was analyzed by real-time RT-qPCR using GoTaq qPCR Master Mix (Promega, Madison, WI, USA) in ABI 7300 (Applied Biosystems, Waltham, MA, USA). Values were normalized to the expression of Gapdh, and the fold difference in expression relative to that of Gapdh is shown. The following primer sets were used: human S100A8 (forward: 5'-GGGATGACCTGAAGAAATTGCTA-3', reverse: 5'TGTTGATATCCAACTCTTTGAACCA-3'), human GAPDH (forward: 5'CATGTTCGTCATGGGGTGAACCA-3', reverse: 5'AGTGATGGCATGGACTGTGGTCAT-3’).

Immunohistochemistry

After deparaffinization of paraffin-fixed tissue, antigen retrieval was performed with autoclaving for $15 \mathrm{~min}$ at $125^{\circ} \mathrm{C}$ in an ethylenediaminetetraacetate buffer solution ( $\mathrm{pH} 9$ ). Endogenous peroxidase activity was blocked using REAL Peroxidase Blocking Reagent (Dako, Carpinteria, CA, USA). Sections were allowed to react overnight at $4^{\circ} \mathrm{C}$ with S100A8 antibody (1:2500; Novus Biologicals, Littleton, CO, USA), RAGE Antibody (1:2000; Sino Biological, Beijing, China), TLR4 antibody (1:4000; Santa Cruz Biotechnology, Dallas, TX, USA), or IL-1 $\beta$ antibody (1:4000; Bioss Antibodies, Woburn, MA, USA). After incubation with Histofine Simple Stain MAX PO (R) (Nichirei, Tokyo, Japan), slides were developed using 3,3-diaminobenzidine as the chromogen.

Histological analysis

Positive cell numbers were assessed in three regions at random points with 500 highpower fields. The sum of the positive cell numbers was used as the score for each individual case.

Immunofluorescence 
After deparaffinization of paraffin-fixed tissue, antigen retrieval was performed with autoclaving for $15 \mathrm{~min}$ at $125^{\circ} \mathrm{C}$ in an ethylenediaminetetraacetate buffer solution $(\mathrm{pH}$ 9). Sections were blocked with 1\% BSA in PBS and were reacted with S100A8/MRP8 Antibody (1:100, LS-B1618-50; LS Bio, Seattle, WA, USA) or MBP Antibody (1:100, bs-0380R; Bioss Antibodies) at $4^{\circ} \mathrm{C}$ overnight. After incubation with Alexa Fluor 488 goat anti-rabbit IgG (cat A11008; Invitrogen) or Alexa Fluor 647 anti-mouse IgG2b (cat A21242; Invitrogen) for $3 \mathrm{~h}$ at room temperature, and 4',6-diamidino-2phenylindole (DAPI) (Vector Laboratories, Burlingame, CA, USA) was used for nuclear counterstaining.

Human primary cell culture

Human nasal epithelial cells (HNEpCs) (PromoCell, Heidelberg, Germany) were cultured at $37^{\circ} \mathrm{C}$ and $5 \% \mathrm{CO}_{2}$ using an airway epithelial cell growth medium with supplements (PromoCell).

Isolation of eosinophils, neutrophils or peripheral blood mononuclear cells (PBMCs)

Eosinophils from healthy peripheral blood were isolated by negative selection using a MACS Isolation Kit (Miltenyi Biotec, Tokyo, Japan). Neutrophils and PBMCs were isolated with the Polymorphprep ${ }^{\mathrm{TM}}$ protocol (Abbott Diagnostics Technologies AS, Oslo, Norway).

Western blot

HNEpCs were lysed with buffer containing protease inhibitor. Eosinophils, neutrophils, and PBMCs were isolated from the peripheral blood of a healthy individual and lysed with buffer containing protease inhibitor. TLR4, RAGE, and S100A8 in the cell lysates were detected by western blotting. For electrophoresis, lysates were boiled for $5 \mathrm{~min}$ in sodium dodecyl sulfate (SDS) polyacrylamide gel electrophoresis sample buffer containing $0.125 \mathrm{mM}$ Tris- $\mathrm{HCl}(\mathrm{pH} 6.8), 20 \%$ glycerol, 4\% SDS, and 10\% 2mercaptoethanol, and loaded onto Next Page GLX-1F5B gels (Gellex International, Tokyo, Japan). Proteins were transferred to membranes using the Trans-Blot Turbo system (Bio-Rad, Hercules, CA, USA). Membranes were blocked for $1 \mathrm{~h}$ at room 
temperature in Blocking-One buffer (Nacalai Tesque, Tokyo, Japan). After blocking, membranes were incubated with anti-RAGE antibody (\#42544, 1:1000; Cell Signaling Technology Japan, Tokyo, Japan), TLR4 antibody (\#sc-293072, 1:200; Santa Cruz Biotechnology) or S100A8/MRP8 Antibody (\#LS-B1618-50, 1:100; LS Bio, Seattle, WA, USA) at $4{ }^{\circ} \mathrm{C}$ overnight, and reacted with anti-rabbit IgG antibody or anti-mouse IgG antibody for $1 \mathrm{~h}$ at room temperature. The signal was then developed with Chemi-Lumi One (Nacalai Tesque).

Human nasal epithelial cells

HNEpCs were treated for $24 \mathrm{~h}$ with recombinant S100A1 $(10 \mu \mathrm{g} / \mathrm{ml})$ (R\&D Systems, Minneapolis, MN, USA), recombinant S100A3 (10 $\mu \mathrm{g} / \mathrm{ml})$ (Novus Biologicals, Littleton, CO, USA), or recombinant S100A9 (10 $\mu \mathrm{g} / \mathrm{ml})$ (R\&D Systems). Human recombinant S100A8 (R\&D Systems) was used for stimulation at a final concentration of $1 \mu \mathrm{g} / \mathrm{ml}$ or $10 \mu \mathrm{g} / \mathrm{ml}$. Supernatants were collected and preserved at $-80^{\circ} \mathrm{C}$.

Enzyme-linked immunosorbent assay (ELISA) and flow cytometric bead array

Cytokine levels in HNEpC culture supernatants were measured with a Cytometric Bead Array Human IL-1 $\beta$ Flex Set (BD Bioscience, Tokyo, Japan) or Human TNF- $\alpha$ Duoset ELISA (R\&D Systems).

All experiments were performed in accordance with relevant guidelines and regulations.

Statistical analysis

All statistical analyses were performed with Prism version 7 (GraphPad Software, San Diego, CA, USA). Data were expressed as means \pm SD. Comparisons between two groups were performed using Student's t-test, and $\mathrm{p}<0.05$ was considered to indicate statistical significance.

\section{RESULTS}

Upregulated genes in nasal polyps from patients with ECRS

In order to investigate the factors involved in the pathogenesis of ECRS, we first performed RNA sequence analysis of nasal polyps obtained from patients with ECRS or NECRS. S100A8 was identified as a significantly upregulated gene in nasal polyps associated with ECRS (Table 1). The expression of other S100 protein-related genes is 
shown in Table 2. S100A9 was significantly upregulated in nasal polyps from patients with ECRS, while $S 100 A 1$ and $S 100 B$ were significantly upregulated in nasal polyps from patients with NECRS. Therefore, we hypothesized that $S 100 A 8$ plays an important role in the pathogenesis of ECRS. We evaluated the expression of S100A8 in locally inflamed tissue of patients with ECRS.

Evaluation of S100A8 expression in nasal polyps associated with ECRS

First, we confirmed that the relative expression of S100A8 measured by RT-qPCR was slightly higher in ECRS than in NECRS, but not significantly (Figure 1A). In immunohistochemistry studies, anti-S100A 8 antibody staining of eosinophils that had infiltrated nasal polyps was stronger in patients with ECRS than in those with NECRS (Figure 1B-D). The number of cells stained with S100A8 was significantly higher in ECRS than in NECRS (Figure 1E, F). These results indicate that the increase in S100A8 in nasal polyps associated with ECRS may be due to infiltrating eosinophils. We also confirmed the expression of S100A8 in peripheral blood eosinophils, neutrophils, and mononuclear cells by western blotting (Supplemental Figure 1).

Expression of the receptor for S100A8 in human nasal epithelial cells

To further investigate how S100A8 functionally contributes to ECRS, we first confirmed the expression of TLR4 and RAGE in HNEpCs using western blotting. Both TLR4 and RAGE were expressed in HNEpCs in the steady state (Figure 2A). Next, the expression of TLR4 and RAGE were compared by immunohistochemistry in ECRS and NECRS. Interestingly, the expression of TLR4 was significantly higher in ECRS than in NECRS (Figure 2B). The expression of RAGE was nonsignificantly higher in ECRS than NECRS (Figure 2C).

S100A8 protein pathophysiology in nasal epithelial cells

Previous reports suggested that various cells promote proinflammatory cytokine production via S100A8 stimulation. Thus, we examined cytokine production by nasal epithelial cells following stimulation with recombinant S100A8 protein for 24 or $48 \mathrm{~h}$. Interestingly, S100A8 induced interleukin-1 beta (IL-1 $\beta$ ) production in nasal epithelial cells (Figure 3A) in a dose-dependent manner (Figure 3B). However, stimulation by S100A8 did not induce interferon-gamma production (Figure 3C). Next, we examined cytokine production by nasal epithelial cells following stimulation with recombinant S100A1, S100A9, and S100A3 proteins as negative controls. S100A8 induced IL-1 $\beta$ 
production in nasal epithelial cells more strongly than S100A1 or S100A3. S100A9 induced IL-1 $\beta$ production as strongly as S100A8 (Figure 3D). In immunohistochemistry, IL-1 $\beta$ was more highly expressed in ECRS than in NECRS, but not significantly (Supplemental Figure 2).

\section{DISCUSSION}

In this study, we demonstrated the clinical implications of S100A8 in ECRS, a refractory disease involving type 2 inflammation. Some previous reports suggested that DAMPs released by damaged tissue might contribute to the exacerbation of allergic diseases ${ }^{8}$. S100A8, a type of DAMP, is produced mainly by blood cells ${ }^{15}{ }^{16}{ }^{17}$. It has been reported that RAGE and TLR4 are receptors for S100A8, and S100A8 induces inflammation by binding to these receptors. Thus, S100A8 is closely related to allergic dermatitis and autoimmune diseases, but its role in ECRS has not been fully explored ${ }^{18}$ 19.

RNA sequencing showed that $S 100 A 8$ was more highly expressed in nasal polyps associated with ECRS than those associated with NECRS. Among other genes in the S100 protein family, S100A9 was also significantly upregulated in nasal polyps associated with ECRS. Previous studies used RNA sequencing to compare gene expression in nasal polyps associated with ECRS and NECRS ${ }^{20}$. In RT-qPCR in this study, there was no significant difference in S100A8 expression between ECRS and NECRS, but expression was nonsignificantly higher in ECRS. However, we believe that the lack of significant difference between ECRS and NECRS is due to the small number of cases examined by RT-qPCR.

However, there are no studies that evaluated the expression of S100 family proteins in local tissues from patients with ECRS. It has been reported that S100A8 is mainly secreted by neutrophils and monocytes ${ }^{15} 1617$. Thus, increased S100A8 gene expression in nasal polyps associated with ECRS might be due to the infiltration of various immune cells. In this study, histological evaluation revealed S100A8 protein expression in tissue-infiltrating eosinophils. No previous reports have described the contribution of eosinophils to the production of S100A8; this finding is new. Further studies will be needed to elucidate the factors that induce the production of S100A8 in eosinophils.

We evaluated the role of S100A8 in the exacerbation of ECRS. Nasal epithelial cells were predicted as a target of S100A8 action. Therefore, we assessed the receptors 
for S100A8 in the nasal epithelium. Previous reports have shown the expression of RAGE and TLR4 in lower airway epithelial cells ${ }^{18} 19$. This study showed that both receptors are expressed in the upper airway as well. In addition, S100A8 has been reported to contribute to the production of various proinflammatory cytokines and increased production of MUC5 AC ${ }^{11}$. Hence, we stimulated nasal epithelial cells with S100A8 and found increased production of IL-1 $\beta$, an important factor for both type 2 and type 3 inflammation. These results are consistent with recent studies showing that severe eosinophilic inflammation is caused by inflammation mediated both by type 2 and type 3 cytokines 45212223 .

We also showed that the expression of TLR4 was higher in ECRS than in NECRS. This result suggests that S100A8 may be highly expressed in ECRS due to the higher expression of TLR4 in ECRS.

This study has some limitations. We did not investigate whether IL-1 $\beta$ production by nasal epithelial cells is mediated by TLR4 or RAGE receptors. Additionally, in immunohistochemistry, the expression of IL- $1 \beta$ was nonsignificantly higher in ECRS than NECRS.

However, the study has three novel features. Comprehensive analysis revealed that $S 100 A 8$ is upregulated in nasal tissues of patients with ECRS. S100A8 protein expression was upregulated in eosinophils infiltrating nasal polyps in ECRS and enhances IL-1 $\beta$ production in upper airway epithelial cells. S100A8 worsens allergic inflammation, implying that S100A8 blockade could promote recovery from ECRS. Therefore, studies with mouse models, as well as clinical tests of S100A8-blocking therapies, should be pursued in the future.

In summary, we found increased expression of $S 100 A 8$ gene expression in nasal polyps from patients with ECRS. S100A8 might be derived from eosinophils that have infiltrated nasal tissue. We also demonstrated that S100A8 enhances IL-1 $\beta$ production in nasal epithelial cells, which may be involved in the pathogenesis of ECRS.

\section{References}

1 Lou, H., Zhang, N., Bachert, C. \& Zhang, L. Highlights of eosinophilic chronic 
rhinosinusitis with nasal polyps in definition, prognosis, and advancement. Int Forum Allergy Rhinol 8, 1218-1225, doi:10.1002/alr.22214 (2018). Tokunaga, T. et al. Novel scoring system and algorithm for classifying chronic rhinosinusitis: the JESREC Study. Allergy 70, 995-1003, doi:10.1111/all.12644 (2015). Bachert, C., Zhang, N., Hellings, P. W. \& Bousquet, J. Endotype-driven care pathways in patients with chronic rhinosinusitis. J Allergy Clin Immunol 141, 1543-1551, doi:10.1016/j.jaci.2018.03.004 (2018). Turner, J. H., Chandra, R. K., Li, P., Bonnet, K. \& Schlundt, D. G. Identification of clinically relevant chronic rhinosinusitis endotypes using cluster analysis of mucus cytokines. J Allergy Clin Immunol 141, 1895-1897 e1897, doi:10.1016/j.jaci.2018.02.002 (2018).

Tomassen, P. et al. Inflammatory endotypes of chronic rhinosinusitis based on cluster analysis of biomarkers. J Allergy Clin Immunol 137, 1449-1456 e1444, doi:10.1016/j.jaci.2015.12.1324 (2016).

6 Tsuda, T. et al. Eosinophil-derived neurotoxin enhances airway remodeling in eosinophilic chronic rhinosinusitis and correlates with disease severity. Int Immunol 31, 33-40, doi:10.1093/intimm/dxy061 (2019).

7 Tsuda, T. et al. Pathological and therapeutic implications of eosinophil-derived semaphorin 4D in eosinophilic chronic rhinosinusitis. J Allergy Clin Immunol 145, 843854 e844, doi:10.1016/j.jaci.2019.12.893 (2020).

8 Paris, G., Pozharskaya, T., Asempa, T. \& Lane, A. P. Damage-associated molecular patterns stimulate interleukin-33 expression in nasal polyp epithelial cells. Int Forum Allergy Rhinol 4, 15-21, doi:10.1002/alr.21237 (2014).

9 Kligman, D. \& Hilt, D. C. The S100 protein family. Trends Biochem Sci 13, 437-443, doi:10.1016/0968-0004(88)90218-6 (1988).

10 Koy, M. et al. Recombinant bovine S100A8 and A9 enhance IL-1beta secretion of interferon-gamma primed monocytes. Vet Immunol Immunopathol 155, 162-170, doi:10.1016/j.vetimm.2013.07.002 (2013).

11 Kang, J. H., Hwang, S. M. \& Chung, I. Y. S100A8, S100A9 and S100A12 activate airway epithelial cells to produce MUC5AC via extracellular signal-regulated kinase and nuclear factor-kappaB pathways. Immunology 144, 79-90, doi:10.1111/imm.12352 (2015).

12 Pham, D. L. et al. Serum S100A8 and S100A9 Enhance Innate Immune Responses in the Pathogenesis of Baker's Asthma. Int Arch Allergy Immunol 168, 138-146, doi:10.1159/000441678 (2015).

13 Wang, S. et al. S100A8/A9 in Inflammation. Front Immunol 9, 1298, doi:10.3389/fimmu.2018.01298 (2018). 
14 Fokkens, W. J. et al. European Position Paper on Rhinosinusitis and Nasal Polyps 2020. Rhinology 58, 1-464, doi:10.4193/Rhin20.600 (2020).

15 Kraakman, M. J. et al. Neutrophil-derived S100 calcium-binding proteins A8/A9 promote reticulated thrombocytosis and atherogenesis in diabetes. J Clin Invest 127, 2133-2147, doi:10.1172/JCI92450 (2017).

16 Lim, S. Y., Yuzhalin, A. E., Gordon-Weeks, A. N. \& Muschel, R. J. Tumor-infiltrating monocytes/macrophages promote tumor invasion and migration by upregulating S100A8 and S100A9 expression in cancer cells. Oncogene 35, 5735-5745, doi:10.1038/onc.2016.107 (2016).

17 Sreejit, G. et al. Neutrophil-Derived S100A8/A9 Amplify Granulopoiesis After Myocardial Infarction. Circulation 141, 1080-1094, doi:10.1161/CIRCULATIONAHA.119.043833 (2020).

18 MacRedmond, R. E., Greene, C. M., Dorscheid, D. R., McElvaney, N. G. \& O'Neill, S. J. Epithelial expression of TLR4 is modulated in COPD and by steroids, salmeterol and cigarette smoke. Respir Res 8, 84, doi:10.1186/1465-9921-8-84 (2007).

19 Reynolds, P. R., Wasley, K. M. \& Allison, C. H. Diesel particulate matter induces receptor for advanced glycation end-products (RAGE) expression in pulmonary epithelial cells, and RAGE signaling influences NF-kappaB-mediated inflammation. Environ Health Perspect 119, 332-336, doi:10.1289/ehp.1002520 (2011).

20 Ninomiya, T. et al. Periostin as a novel biomarker for postoperative recurrence of chronic rhinosinitis with nasal polyps. Sci Rep 8, 11450, doi:10.1038/s41598-018-29612-2 (2018).

21 Bal, S. M. et al. IL-1 $\beta$, IL-4 and IL-12 control the fate of group 2 innate lymphoid cells in human airway inflammation in the lungs. Nat Immunol 17, 636-645, doi:10.1038/ni.3444 (2016).

22 Mahmutovic Persson, I. et al. IL-1 $\beta$ mediates lung neutrophilia and IL-33 expression in a mouse model of viral-induced asthma exacerbation. Respir Res 19, 16, doi:10.1186/s12931-018-0725-z (2018).

23 Rossios, C. et al. Sputum transcriptomics reveal upregulation of IL-1 receptor family members in patients with severe asthma. J Allergy Clin Immunol 141, 560-570, doi:10.1016/j.jaci.2017.02.045 (2018).

\section{Figure legend}


Figure 1 S100A8 was expressed in eosinophils that have infiltrated nasal tissue.

A. Gene levels of S100A8 were measured by RT-qPCR and compared $(n=8$ ECRS, $n$ $=4$ NECRS).

B, C. Hematoxylin and eosin staining of nasal tissues and immunohistochemical detection of S100A8. Images are of representative samples from patients with ECRS. Scale bars, $100 \mu \mathrm{m}$.

D. Immunofluorescence staining for S100A8 (green), MBP (red), or DAPI (blue). Scale bars, $100 \mu \mathrm{m}$.

E. Expression of S100A8 in nasal polyps from patients with ECRS or NECRS.

F. Immunohistochemical staining for S100A8; positive cells were counted $(\mathrm{n}=15$ ECRS, $\mathrm{n}=15$ NECRS).

NS, not significant, ${ }^{*} \mathrm{P}<0.05$, Student's t-test. (A), (F). Values are means $\pm \mathrm{SD}$. MBP, major basic protein; DAPI, 4',6-diamidino-2-phenylindole

\section{Figure 2. Expression of S100A8 receptor on HNEpCs.}

A. Western blots showing that both TLR4 and RAGE are expressed in HNEpCs. Fulllength blots/gels are presented in Supplementary Figure 3.

B. Immunohistochemical staining for TLR4; positive cells were counted ( $n=5$ ECRS, $\mathrm{n}=5$ NECRS). Left: representative images for ECRS and NECRS; right: number of positive cells.

C. Immunohistochemical staining for RAGE; positive cells were counted ( $n=5$ ECRS, $\mathrm{n}=5$ NECRS). Left: representative images for ECRS and NECRS; right: number of positive cells.

NS, not significant. $* \mathrm{P}<0.05$, Student's t-test (B), (C). Values are means $\pm \mathrm{SD}$.

TLR4, Toll-like receptor 4; RAGE, receptor for advanced glycation end products; HNEpCs, human nasal epithelial cells. 
Figure 3. IL-1 $\beta$ production by HNEpCs stimulated with recombinant S100A8 protein.

A. HNEpCs were stimulated with recombinant S100A8 protein $(10 \mu \mathrm{g} / \mathrm{ml})$ with control for 24 or $48 \mathrm{~h}$. IL-1 $\beta$ levels in culture supernatant were determined using CBA.

B. HNEpCs were stimulated with recombinant S100A8 protein $(1 \mu \mathrm{g} / \mathrm{ml}$ or $10 \mu \mathrm{g} / \mathrm{ml})$ with control for $24 \mathrm{~h}$. IL-1 $\beta$ levels in culture supernatant were determined using CBA.

C. HNEpCs were stimulated with recombinant S100A8 protein $(10 \mu \mathrm{g} / \mathrm{ml})$ with control for $24 \mathrm{~h}$. INF- $\gamma$ levels in culture supernatant were determined using ELISA.

D. HNEpCs were stimulated with recombinant S100A1 protein $(10 \mu \mathrm{g} / \mathrm{ml})$, recombinant S100A3 protein $(10 \mu \mathrm{g} / \mathrm{ml})$, recombinant S100A8 protein $(10 \mu \mathrm{g} / \mathrm{ml})$, or recombinant S100A9 protein $(10 \mu \mathrm{g} / \mathrm{ml})$ with control for $24 \mathrm{~h}$. IL-1 $\beta$ levels in culture supernatant were determined using CBA.

NS, not significant, $* * \mathrm{P}<0.01, * * * \mathrm{P}<0.005$ Student's t-test (A), (B), (C), (D). Values are means $\pm \mathrm{SD}$.

HNEpCs, human nasal epithelial cells; IL-1 $\beta$, interleukin-1 beta; CBA, cytometric bead array; Ctrl, control. 
Table 1. Gene expression profile of nasal polyps from patients with eosinophilic chronic rhinosinusitis (ECRS) versus non-eosinophilic chronic rhinosinusitis (NECRS).

\begin{tabular}{|l|l|l|}
\hline mRNA & Fold change & $\mathrm{P}$ \\
\hline CLC & 470.1 & 0.001 \\
\hline TNFAIP8L2-SCNM1 & 380.5 & 0.013 \\
\hline S100A8 & 313.0 & 0.047 \\
\hline
\end{tabular}


Table 2. S100 family protein gene expression in nasal polyps from patients with eosinophilic chronic rhinosinusitis (ECRS) versus non-eosinophilic chronic rhinosinusitis (NECRS).

\begin{tabular}{|c|c|c|}
\hline mRNA & Fold change & $\mathrm{P}$ \\
\hline S100A1 & 4.357 & 0.030 \\
\hline$S 100 A 2$ & -10.813 & 0.472 \\
\hline$S 100 A 3$ & -1.489 & 0.885 \\
\hline$S 100 A 4$ & -1.238 & 0.710 \\
\hline S100A5 & -2.106 & 0.811 \\
\hline S100A6 & -1.824 & 0.236 \\
\hline S100A7 & 3.924 & 0.423 \\
\hline$S 100 A 7 A$ & -3.347 & 0.196 \\
\hline S100A7L2 & 1.000 & Not a number \\
\hline$S 100 A 8$ & -313.013 & 0.047 \\
\hline S100A9 & -13.014 & 0.031 \\
\hline S100A10 & -1.637 & 0.112 \\
\hline S100A11 & -2.158 & 0.051 \\
\hline$S 100 A 12$ & -3.444 & 0.708 \\
\hline$S 100 A 13$ & -2.122 & 0.223 \\
\hline S100A14 & 1.195 & 0.654 \\
\hline S100A16 & 2.314 & 0.125 \\
\hline
\end{tabular}




\begin{tabular}{|l|l|l|}
\hline S100B & 111.508 & 0.010 \\
\hline S100G & 1.000 & Not a number \\
\hline S100P & -4.583 & 0.100 \\
\hline S100PBP & 1.395 & 0.733 \\
\hline S100Z & -17.283 & 0.059 \\
\hline
\end{tabular}




\section{Figures}

\section{Figure 1}

S100A8 was expressed in eosinophils that have infiltrated nasal tissue. A. Gene levels of S100A8 were measured by RT-qPCR and compared ( $n=8$ ECRS, $n=4$ NECRS). B, C. Hematoxylin and eosin staining of nasal tissues and immunohistochemical detection of S100A8. Images are of representative samples from patients with ECRS. Scale bars, $100 \mu \mathrm{m}$. D. Immunofluorescence staining for S100A8 (green), MBP (red), or DAPI (blue). Scale bars, $100 \mu \mathrm{m}$. E. Expression of S100A8 in nasal polyps from patients with ECRS or NECRS. F. Immunohistochemical staining for S100A8; positive cells were counted ( $n=15 \mathrm{ECRS}, \mathrm{n}$ $=15$ NECRS). NS, not significant, ${ }^{*} P<0.05$, Student's t-test. (A), (F). Values are means \pm SD. MBP, major basic protein; DAPI, 4',6-diamidino-2-phenylindole

\section{Figure 2}

Expression of S100A8 receptor on HNEpCs. A. Western blots showing that both TLR4 and RAGE are expressed in HNEpCs. Full length blots/gels are presented in Supplementary Figure 3. B. Immunohistochemical staining for TLR4; positive cells were counted ( $n=5$ ECRS, $n=5$ NECRS). Left: representative images for ECRS and NECRS; right: number of positive cells. C. Immunohistochemical staining for RAGE; positive cells were counted ( $n=5$ ECRS, $n=5$ NECRS). Left: representative images for ECRS and NECRS; right: number of positive cells. NS, not significant. ${ }^{*} \mathrm{P}<0.05$, Student's t-test (B), (C). Values are means \pm SD. TLR4, Toll-like receptor 4; RAGE, receptor for advanced glycation end products; HNEpCs, human nasal epithelial cells.

\section{Figure 3}

IL-1 $\beta$ production by HNEpCs stimulated with recombinant S100A8 protein. A. HNEpCs were stimulated with recombinant S100A8 protein $(10 \mu \mathrm{g} / \mathrm{ml})$ with control for 24 or $48 \mathrm{~h}$. IL-1 $\beta$ levels in culture supernatant were determined using CBA. B. HNEpCs were stimulated with recombinant S100A8 protein (1 $\mu \mathrm{g} / \mathrm{ml}$ or $10 \mu \mathrm{g} / \mathrm{ml}$ ) with control for $24 \mathrm{~h}$. IL-1 $\beta$ levels in culture supernatant were determined using CBA. C. HNEpCs were stimulated with recombinant S100A8 protein $(10 \mu \mathrm{g} / \mathrm{ml})$ with control for $24 \mathrm{~h}$. INF- $\gamma$ levels in culture supernatant were determined using ELISA. D. HNEpCs were stimulated with recombinant S100A1 protein $(10 \mu \mathrm{g} / \mathrm{ml})$, recombinant S100A3 protein $(10 \mu \mathrm{g} / \mathrm{ml})$, recombinant S100A8 protein (10 $\mu \mathrm{g} / \mathrm{ml})$, or recombinant S100A9 protein $(10 \mu \mathrm{g} / \mathrm{ml})$ with control for $24 \mathrm{~h}$. IL-1 $\beta$ levels in culture supernatant were determined using CBA. NS, not significant, ${ }^{\star \star} \mathrm{P}<0.01$, ${ }^{\star \star \star} \mathrm{P}<0.005$ Student's t-test (A), (B), (C), (D). Values are means \pm SD. HNEpCs, human nasal epithelial cells; IL-1 $\beta$, interleukin-1 beta; CBA, cytometric bead array; Ctrl, control. 


\section{Supplementary Files}

This is a list of supplementary files associated with this preprint. Click to download.

- SupplementalFigurefileVer2.pdf 